\title{
Neural Adaptive Observer Based Fault Detection and Identification for Satellite Attitude Control Systems
}

\author{
Qing Wu Mehrdad Saif* \\ School of Engineering Science \\ Simon Fraser University \\ Vancouver, V5A 1S6, Canada
}

\begin{abstract}
A neural adaptive observer (NAO) based fault detection and identification (FDI) strategy for a class of nonlinear systems is presented in this paper. The observer input is designed in a structure similar to feedback neural networks. The parameters in the NAO input are updated by using the Extended Kalman Filter (EKF) algorithm. The convergence of the learning process is analyzed in terms of a quadratic Lyapunov function. Moreover, stability of the observer input and the NAO-based system are investigated respectively. Finally, the proposed FDI strategy is applied to a micro-satellite attitude control system. Several simulation results demonstrate that the NAO based FDI method can detect and specify both abrupt and incipient faults with satisfactory performance.
\end{abstract}

\section{INTRODUCTION}

Maturing space technologies and growing mission demands have lead to building more sophisticated spacecraft such as satellites and space shuttles. During the operation of a satellite, three-axis attitude control strategy is usually applied to keep the satellite from various environmental disturbances and inner moment-of-inertia variations. However, increasing complexity of the satellite inevitably raises the probability of faults which are characterized by the unpermitted deviation of at least one characteristic property of the system component, actuator or sensor, from the acceptable condition. The consequences of faults are serious for the satellite from the viewpoints of cost and risk. Therefore, there is a growing need for onboard fault detection and identification for a satellite attitude control systems to enhance its reliability and autonomy.

In the last two decades, fault detection and identification for linear and nonlinear systems have received a great deal of attention. The work have been summarized in [1], [2], [3], [4], and [5]. With respect to the methodologies, the most frequently used methods in the process fault detection are based on system estimation, for example, system identification and observer-based approaches [6], [7] and [8].

In recent years, researchers have focused on the fault detection and identification for nonlinear systems. Traditionally, the FDI issues for a nonlinear dynamic system entail linearizing the model at an operating point firstly, and then applying well-known FDI methods for linear systems to generate a residual, which is compared with a threshold

*Corresponding author: saif@cs.sfu.ca function to determine whether a fault has occurred or not. This kind of approach is of much limitation, because an unsuitable choice of the threshold function may cause false fault alarms. To deal with systems with inherent nonlinearity, direct nonlinear models are needed. The original inspiration of using neural networks $(\mathrm{NN})$ in nonlinear FDI is based on the property that neural networks can approximate any arbitrary nonlinear function under certain conditions [9]. NN or (NN-like) model based nonlinear fault detection methods have been studied for several years, i.e. [10], [11], [12], [13] and [14]. However, the key point of applying neural networks or NN-like model to the FDI of a practical system is that this model must be able to approximate the system or faults with sufficient accuracy and satisfy the real time requirement.

In this paper, a neural adaptive observer based fault detection and identification approach is proposed and applied to a satellite attitude control system. The convergence of the parameters update law and the stability of the NAO based system are investigated. The advantage of this NAO based fault detection and identification approach is that it can detect and identify both abrupt and incipient faults with high accuracy.

\section{PROBLEM STATEMENT}

Considering a class of nonlinear systems represented by

$$
\begin{aligned}
& \dot{x}(t)=f(x(t))+g(x(t)) u(t)+f_{a}(x, u) \\
& y(t)=h(x(t))
\end{aligned}
$$

where $x \in \mathfrak{R}^{n}$ is the state vector, $u \in \mathfrak{R}^{m}$ is the input vector and $y \in \mathfrak{R}^{r}$ is the measurable output vector of the system, respectively. The state function $f: \mathfrak{R}^{n} \rightarrow \mathfrak{R}^{n}$, input function $g: \mathfrak{R}^{n} \rightarrow \mathfrak{R}^{n \times m}$ and output function $h: \mathfrak{R}^{n} \rightarrow \mathfrak{R}^{r}$ are all smooth vector fields. $f_{a}(x, u)$ : $\mathfrak{R}^{n} \times \mathfrak{R}^{m} \rightarrow \mathfrak{R}^{n}$ is the fault vector, whose elements denote system component faults as well as actuator faults. It should be noted that $f_{a}(x, u)$ in (1) does not characterize sensor faults. The description of a sensor fault entails an additional term in the output equation. In general, it is unreasonable to make further assumptions about the characteristics of a fault but consider that as an uncertain nonlinear time function of the system state and input.

Throughout this paper, following assumptions are introduced to make it convenient to analyze the stability of the NAO based system. 
Assumption 1: The states in $x(t)$ are all available for measurement.

Assumption 2: The state function $f(x(t))$ is differentiable at $\hat{x}$, which is

$$
A(t)=\left.\frac{\partial f}{\partial x}\right|_{x=\hat{x}}
$$

where $A(t)$ is an $n \times n$ matrix. So, following equation can be derived through series expansion of $f(x)$ at $\hat{x}$.

$$
f(x)-f(\hat{x})=A(t) \tilde{x}(t)+\phi(\hat{x}, x)
$$

where $\phi(\hat{x}, x)=o(\|\tilde{x}(t)\|)$, which contains the high order term of the state estimation error $\tilde{x}(t)=x(t)-\hat{x}(t)$.

Assumption 3: Input function $g(x)$ and output function $h(x)$ are Lipschitz in $x$ with Lipschitz constants $l_{g}$ and $l_{h}$.

$$
\begin{aligned}
\|g(x)-g(\hat{x})\| & \leq l_{g}\|\tilde{x}(t)\| \\
\|h(x)-h(\hat{x})\| & \leq l_{h}\|\tilde{x}(t)\|
\end{aligned}
$$

Assumption 4: System input vector is bounded by $l_{u}$ and fault $f_{a}(x, u)$ is bounded by $f_{m}$.

Assumption 5: There is a real number $l_{\phi}>0$ such that $\phi$ is bounded via

$$
\|\phi(\hat{x}, x)\| \leq l_{\phi}\|\tilde{x}(t)\|
$$

Assumption 6: Symmetric matrix $\Gamma(t)$ has the following property:

$$
\beta_{1} I_{n} \leq \Gamma(t) \leq \beta_{2} I_{n}
$$

where $\beta_{1}, \beta_{2}$ are positive real numbers, and $\Gamma(t)$ is the solution of the following Lyapunov equation:

$$
A^{\top}(t) \Gamma(t)+\Gamma(t) A(t)+\dot{\Gamma}(t)=-Q
$$

where $Q$ is a symmetric positive definite matrix.

The objective of this paper is to develop a NAO based fault detection and identification strategy for the nonlinear dynamic system (1). The NAO is updated iteratively by the observer input, which is denoted as $M(t)$, so that it is able to detect the deviation in the system dynamics caused by faults. On one hand, $M(t)$ needs time to adjust its parameters to track the fault in the transient process. Corresponding residuals are generated to diagnose the fault. On the other hand, $M(t)$ itself is able to track the fault quickly with sufficiently high accuracy so as to specify the characteristics of a variety of faults.

\section{NEURAL ADAPTIVE OBSERVER BASED FDI}

\section{A. Design of Neural Adaptive Observer}

In this section, a neural adaptive observer for the purpose of fault detection and identification of a class of nonlinear systems described by (1) is discussed. As for the nonlinear system (1), in practice, it is always possible to measure the input and output vector rather than the state vector. In this case, only system input and output are able to be used to design the observer for fault detection. Since a fault in the system is of uncertainty and nonlinearity, and neural networks can approximate arbitrary nonlinear functions under certain conditions, it is natural to apply neural networks to the fault detection of nonlinear system. Unlike directly using neural network to be a model of the system, a neural adaptive observer using only the the system input and output is constructed as follows.

$$
\begin{aligned}
\dot{\hat{x}}(t) & =f(\hat{x}(t))+g(\hat{x}(t)) u(t)+M(t) \\
\hat{y}(t) & =h(\hat{x}(t)) \\
M_{i}(t) & =W_{i}(t) \sigma\left(V_{i}(t) I_{i}(t)\right)
\end{aligned}
$$

where $\hat{x}(t)$ is the observer state vector at the $t$ th time. $M_{i}(t)$, for $i=1, \cdots, n$ is the $i$ th element of the observer input vector $M(t) . W_{i}(t)$ and $V_{i}(t)=\left[V_{i, 1}(t), \cdots, V_{i, p+q}(t)\right]$ are the parameters in the $i$ th NAO input at the $t$ th time. $I_{i}(t)=$ $\left[M_{i}(t-\tau), \cdots, M_{i}(t-p \tau), \tilde{y}_{i}(t-\tau), \cdots, \tilde{y}_{i}(t-q \tau)\right]^{\top}$, where $\tau$ denotes the sampling time interval or step length. $\tilde{y}_{i}(t)$ is the $i$ th element of the output estimation error $\tilde{y}(t)=h(x(t))-h(\hat{x}(t)) \cdot \sigma(\cdot)$ is a sigmoid-type activation function, which is always tansig and logsig etc. in the neural network terminology. In this study, the activation function is chosen as $\sigma(x)=\left(1-\mathrm{e}^{-x}\right) /\left(1+\mathrm{e}^{-x}\right)$, whose smooth non-saturation activation range is wider than the $\tanh (x)$. Suitable $p$ and $q$ are selected based on the time delay of practical systems and real time requirement. Large $p$ and $q$, though guaranteeing the convergence of training, may take more computation time and cause unnecessary time delay.

The observer input are recursively updated by previous $p$ step observer inputs from $M(k-\tau)$ to $M(k-p \tau)$ and previous $q$ step system output error from $\tilde{y}(t-\tau)$ to $\tilde{y}(t-$ $q \tau)$, which is shown in (4).

Subtracting the observer equation (4) from the system equation (1), and according to (2), the state estimation error $\tilde{x}(t)$ is derived to be:

$$
\begin{aligned}
\dot{\tilde{x}}(t)= & A(t) \tilde{x}(t)+\phi(\hat{x}, x)+(g(x)-g(\hat{x})) u(t) \\
& +f_{a}(t)-M(t)
\end{aligned}
$$

Remark 1: When the nonlinear system is of no fault, which means $f_{a}(t)=0$, the ideal observer state is identical to the real system state. $M(t)$ is equal to or close to zero correspondingly. When a fault occurs, $M(t)$ is expected to approach $f_{a}(t)$ as soon as possible. Thus, the observer input is a nonlinear estimator of the fault $f_{a}(t)$.

\section{B. Update Law and Its Convergence}

Similar to general multilayer neural networks, the parameters in the NAO can be learned iteratively by using various optimization methods, such as backpropagation algorithm [15], and Levenberg-Marquardt algorithm [16], etc. In this study, the Extended Kalman Filter (EKF) algorithm [17] is adopted to update the NAO parameters so as to guarantee a fast convergence rate. Let us consider the parameters updating of the $i$ th element of the NAO. The parameters are defined as

$$
\theta_{i}(k)=\left[W_{i}(k), V_{i, 1}(k), \cdots, V_{i, p+q}(k)\right]^{\top}
$$


where $k$ denotes sampling time. The relationship between $k$ and $t$ is $k=t / \tau$. The parameters are updated at each sampling time based on the following rules:

$$
\begin{aligned}
\theta_{i}(k) & =\theta_{i}(k-1)+\eta_{i} K_{i}(k)\left[y_{i}(k)-\hat{y}_{i}(k)\right] \\
K_{i}(k) & =P_{i}(k) H_{i}(k)\left[H_{i}(k)^{\top} P_{i}(k) H_{i}(k)+R_{i}(k)\right]^{-1} \\
P_{i}(k+1) & =P_{i}(k)-K_{i}(k) H_{i}(k)^{\top} P_{i}(k)
\end{aligned}
$$

where $\eta_{i}$ is the learning factor. $K_{i}(k)$ is known as the Kalman gain, $P_{i}(k)$ is the covariance matrix of the state estimation error, and $R_{i}(k)$ is the estimated covariance matrix of noise. Here, $R_{i}(k)$ is recursively calculated according to $[18]$ :

$$
R_{i}(k)=R_{i}(k-1)+\left[\tilde{y}_{i}(k)^{2}-R_{i}(k-1)\right] / k
$$

$H_{i}(k)$ is the derivative of $\hat{y}_{i}(k)$ with respect to $\theta_{i}$. Based on the observer input in (4), $H_{i}(k)$ is expressed as:

$$
\begin{aligned}
& H_{i}(k)=\left.\frac{\partial \hat{y}_{i}(k)}{\partial \theta_{i}}\right|_{\theta_{i}=\theta_{i}(k-1)} \\
& = \begin{cases}h^{\prime} \cdot \sigma\left(Z_{i}(k)\right) & \theta_{i}=W_{i} \\
h^{\prime} \cdot W_{i}(k) M_{i}(k-j) \sigma^{\prime}\left(Z_{i}(k)\right) & \theta_{i}=V_{i, j} \\
h^{\prime} \cdot W_{i}(k) \tilde{y}_{i}(k-j) \sigma^{\prime}\left(Z_{i}(k)\right) & \theta_{i}=V_{i, p+j}\end{cases}
\end{aligned}
$$

where

$$
Z_{i}(k)=\sum_{j=1}^{p} V_{i, j}(k) M_{i}(k-j)+\sum_{j=1}^{q} V_{i, p+j}(k) \tilde{y}_{i}(k-j)
$$

With respect to the convergence of learning process, following theorem is established.

Assumption 7: Since $R_{i}(k)$, for SISO systems represents the estimated variance of noise, it is assumed that $0<$ $R_{i}(k) \ll H_{i}(k)^{\top} P_{i}(k) H_{i}(k)$.

Theorem 1: Consider the nonlinear system (1) and its neural adaptive observer (4), when EKF algorithm is adopted to update the parameters in $M(k)$, if the learning factor satisfies

$$
0<\eta_{i}<2+\frac{2 R_{i}(k)}{\left\|H_{i}(k)\right\|^{2} \lambda_{\max }\left(P_{i}(k)\right)}
$$

then the updating process is convergent.

Proof: For simplicity of presentation, step $k$ is omitted if unnecessary during the following proof. Based on the assumption 7 , if condition (9) satisfies, then

$$
\begin{aligned}
0<\eta_{i} & <2+\frac{2 R_{i}}{H_{i}^{\top} P_{i} H_{i}} \\
& <2 \frac{H_{i}^{\top} P_{i} H_{i}+R_{i}}{H_{i}^{\top} P_{i} H_{i}}
\end{aligned}
$$

Therefore,

$$
0<\eta_{i} H_{i}^{\top} P_{i} H_{i}\left[H_{i}^{\top} P_{i} H_{i}+R_{i}\right]^{-1}<2
$$

That is equivalent to

$$
0<\eta_{i} H_{i}(k)^{\top} K_{i}(k)<2
$$

A Lyapunov function candidate is constructed as follows

$$
V(k)=\frac{1}{2} \tilde{y}_{i}(k)^{2}
$$

The difference of $V(k)$ is

$$
\begin{aligned}
\Delta V(k) & =V(k+1)-V(k) \\
& =\Delta \tilde{y}_{i}(k)\left(\tilde{y}_{i}(k)+\frac{1}{2} \Delta \tilde{y}_{i}(k)\right)
\end{aligned}
$$

The difference of error $\tilde{y}_{i}(k)$ can be approximated to be

$$
\begin{aligned}
\Delta \tilde{y}_{i}(k) & =\left(\frac{\partial \tilde{y}_{i}(k)}{\partial \theta_{i}(k)}\right)^{\top} \Delta \theta_{i}(k) \\
& =-\left(\frac{\partial \hat{y}_{i}(k)}{\partial \theta_{i}(k)}\right)^{\top} \Delta \theta_{i}(k) \\
& =-\eta_{i} H_{i}(k)^{\top} K_{i}(k) \tilde{y}_{i}(k)
\end{aligned}
$$

Therefore, the difference of $V(k)$ is

$$
\begin{aligned}
\Delta V(k) & =\Delta \tilde{y}_{i}(k)\left(\tilde{y}_{i}(k)+\frac{1}{2} \Delta \tilde{y}_{i}(k)\right) \\
& =-\eta_{i} H_{i}^{\top} K_{i}\left(1-\frac{1}{2} \eta_{i} H_{i}^{\top} K_{i}\right) \tilde{y}_{i}(k)^{2}
\end{aligned}
$$

If condition (9) is guaranteed, then, according to (12) and (16), $\Delta V(k)<0$. Thus, the updating process is convergent, if the sufficient condition (9) is guaranteed. That means, $\exists$ time $\kappa_{i}$ and a sufficiently small number $\delta_{i}>0$ such that, for $k>\kappa_{i},\left|f_{a i}(k)-M_{i}(k)\right|<\delta_{i}$. Correspondingly, $\left\|f_{a}(k)-M(k)\right\|<\left(\sum_{i=1}^{n} \delta_{i}^{2}\right)^{1 / 2}=\delta_{f}$.

Remark 2: In order to guarantee the condition in assumption 7 , the initial value of $P_{i}(k)$ is usually chosen as a large identity matrix.

Remark 3: One way to detect fault is to observe the residual which is selected as $\tilde{y}_{i}(k)$. If no faults occur in the system, $\tilde{y}_{i}$ should be zero or close to zero. On the other hand, a nonzero $\tilde{y}_{i}$ larger than a certain threshold would represent the occurrence of a fault.

Remark 4: when above neural adaptive observer is used in the fault detection, the observer input $M(k)$ can specify the fault in the process. This avoids generating residuals and choosing a suitable threshold for a specific fault. The faults which can be identified include component faults and actuator faults. However, to isolate these faults needs additional work.

\section{Stability Analysis}

This section first of all deals with the stability of the NAO input $M_{i}(t)$. Since $M_{i}(t)$ is updated at each discrete time, it can be described as:

$$
\left.M_{i}(k+1)=W_{i} \sigma\left(\sum_{j=1}^{p} V_{i, j} M_{i}(k-j+1)+B_{i}(k)\right) 17\right)
$$

where $B_{i}(k)=\sum_{j=1}^{q} V_{i, p+j} \tilde{y}_{i}(k-j+1)$ is the bias. Defining a vector

$$
z=\left[z_{1}(k), \cdots, z_{p}(k)\right]^{\top}
$$


where $z_{j}(k)=M_{i}(k-j+1)$, for $j=1, \cdots, p$ is a new state vector of the observer input. Thus, the NAO input can be rewritten as

$$
z_{p}(k+1)=W_{i} \sigma\left(\sum_{j=1}^{p} V_{i, j} z_{j}(k)+B_{i}(k)\right)
$$

It is obvious that $z_{p}(k)$ is $\mathrm{BIBO}$ (Bounded Input and Bounded Output) stable, because $\max \left\{z_{p}(k)\right\} \leq \pm W$ as a consequence of the saturation of $\sigma(\cdot)$. The stability of $z_{p}(k)$ can be analyzed in a similar way with that in [19].

Let $z^{*}=\left[z_{1}^{*}, \cdots, z_{p}^{*}\right]$ be an equilibrium point of the equation (18). It is well-known that the equilibrium point of (18) satisfies $z(k+1)=z(k)=z^{*}, k=0,1,2, \ldots$ Due to the recursive property of the state $z$, it is easy to see that the equilibrium state equation may be represented as

$$
z_{p}^{*}=W_{i} \sigma\left(\sum_{j=1}^{p} V_{i, j} z_{j}^{*}+B_{i}\right)
$$

and $z_{1}^{*}=z_{2}^{*}=, \cdots,=z_{p}^{*}$.

Lemma: The system $\vec{x}(t+1)=\Phi \vec{x}(t)$ is asymptotically stable if and only if all the eigenvalues of $\Phi$ are located inside the unit circle in the complex plane [20].

A theorem related to the stability of the equilibrium point of (18) is presented as follows.

Theorem 2: The equilibrium point $z^{*}$ from (18) is asymptotically stable if the absolute value of $W_{i} \cdot \Delta \cdot \sum_{j=1}^{p} V_{i, j}$ is less than one, where $\Delta$ is of the following form

$$
\Delta=\frac{1}{2}\left[1-\sigma^{2}\left(\sum_{j=1}^{p} V_{i, j} z_{p}^{*}+B_{i}\right)\right]
$$

Proof: Take an approximation of $z_{p}(k+1)$ around the equilibrium point $z_{p}^{*}$ by means of Taylor expansion

$$
\begin{aligned}
z_{p}(k+1)= & W_{i} \sigma\left(\sum_{j=1}^{p} V_{i, j} z_{j}(k)+B_{i}\right) \\
\simeq & W_{i} \sigma\left(\sum_{j=1}^{p} V_{i, j} z_{p}^{*}+B_{i}\right) \\
& +W_{i} \cdot \Delta \cdot \sum_{j=1}^{p} V_{i, j} \cdot\left(z_{p}(k)-z_{p}^{*}\right)
\end{aligned}
$$

The first order derivative of $z_{p}(k+1)$ evaluated at the equilibrium point $z_{p}^{*}$ is $W_{i} \cdot \Delta \cdot \sum_{j=1}^{p} V_{i, j}$. The value of $\Delta$ is in the interval $(0,1 / 2)$. Hence, the equilibrium point $z^{*}$ is asymptotically stable if the absolute value of $W_{i} \cdot \Delta \cdot \sum_{j=1}^{p} V_{i, j}$ is less than one. This completes the proof of theorem 2 .

Remark 5: For a healthy system with an ideal observer, $\tilde{y}_{i}$ should be zero. As a result, $M_{i}(k)$ becomes an autonomous system, and Theorem 2 can be directly applied to this case. When a fault occurs, $B_{i}$ in (17) containing $\tilde{y}$ is nonzero. The value of $W_{i} \cdot \Delta \cdot \sum_{j=1}^{p} V_{i, j}$ is thus modified to satisfy the Theorem 2. Therefore, Theorem 2 provides a stability criterion for both healthy and faulty systems. When system is healthy, $M_{i}(k)$ is asymptotically stable to equilibrium point zero. When a fault $f_{a i}(k)$ occurs in the system, $M_{i}(k)$ will be asymptotically stable to the nonzero equilibrium point $f_{a i}(k)$.

As for the stability of the NAO based nonlinear FDI system, following theorem can be obtained.

Theorem 3: Consider the nonlinear system (1) and its NAO described in (4). Let Assumptions 1-6 hold. If following inequality (22) is satisfied, then the state estimation error $\tilde{x}(t)$ is bounded.

$$
\lambda_{\min }(Q)>2 l_{\phi} \beta_{2}+2 l_{g} l_{u} \beta_{2}+\delta_{f}
$$

Proof: Consider the following Lyapunov function candidate

$$
V(\tilde{x}(t), t)=\tilde{x}^{\top}(t) \Gamma(t) \tilde{x}(t)
$$

where $\Gamma(t)$ satisfies Assumption 6 .

The derivative of this Lyapunov function candidate with respect to time $t$ is

$$
\dot{V}(t)=\dot{\tilde{x}}(t)^{\top} \Gamma(t) \tilde{x}(t)+\tilde{x}^{\top}(t) \dot{\Gamma}(t) \tilde{x}(t)+\tilde{x}^{\top}(t) \Gamma(t) \dot{\tilde{x}}(t)
$$

Substituting the state error equation (5) into equation (24), and using the conclusion in the proof of convergence of the parameters updating process, there is

$$
\begin{aligned}
\dot{V}(t)= & \tilde{x}(t)^{\top}\left(A(t)^{\top} \Gamma(t)+\Gamma(t) A(t)+\dot{\Gamma}(t)\right) \tilde{x}(t) \\
& +2 \tilde{x}(t)^{\top} \Gamma(t) \phi+2 \tilde{x}(t)^{\top} \Gamma(t)(g(x)-g(\hat{x})) u(t) \\
& +2 \tilde{x}(t)^{\top} \Gamma(t)\left(f_{a}(t)-M(t)\right) \\
\leq & -\lambda_{\min }(Q)\|\tilde{x}(t)\|^{2}+2 l_{\phi}\|\Gamma(t)\|\|\tilde{x}(t)\|^{2} \\
& +2 l_{g} l_{u}\|e(t)\|^{2}\|\Gamma(t)\|+2\|\tilde{x}(t)\|\|\Gamma(t)\| \delta_{f} \\
\leq & -\lambda_{\min }(Q)\|\tilde{x}(t)\|^{2}+2 l_{\phi}\|\Gamma(t)\|\|\tilde{x}(t)\|^{2} \\
& +2 l_{g} l_{u}\|\tilde{x}(t)\|^{2}\|\Gamma(t)\|+\delta_{f}\left(\|\tilde{x}(t)\|^{2}+\|\Gamma(t)\|^{2}\right) \\
\leq & \left(-\lambda_{\min }(Q)+2 l_{\phi} \beta_{2}+2 l_{g} l_{u} \beta_{2}+\delta_{f}\right)\|\tilde{x}(t)\|^{2}+\delta_{f} \beta_{2}^{2}
\end{aligned}
$$

So, if inequality (22) is satisfied, then

$$
\dot{V} \leq-\gamma\|\tilde{x}(t)\|^{2}+\delta_{f} \beta_{2}^{2}
$$

where

$$
\gamma=\lambda_{\min }(Q)-2 l_{\phi} \beta_{2}-2 l_{g} l_{u} \beta_{2}-\delta_{f}
$$

The above inequality guarantees that $\dot{V}<0$ when $\|\tilde{x}\|>$ $\sqrt{\frac{\delta_{f} \beta_{2}^{2}}{\gamma}}$. Since $\lim _{k \rightarrow \infty} \delta_{f}=0$ and $\beta_{2}$ and $\gamma$ are both finite constant, the state estimation error will converge to a sufficiently small bound as $k \rightarrow \infty$. Therefore, inequality (22) is a sufficient condition to guarantee the boundedness of the state estimation error $\tilde{x}(t)$.

\section{FDI FOR SATELLITE ATTITUDE CONTROL SYSTEMS}

In this section, the proposed NAO based fault detection and identification scheme is applied to a microsatellite attitude control system. Compared with large satellites, microsatellites have many advantages in terms of launch technology, cost, reliability and manufacturing time. So, 
microsatellites, promising space vehicle, play more and more important roles in various space missions, such as remote sensing, climate data collection, Earth observation and data communication. For microsatellites without flexible appendages, it is natural to consider the rigid body dynamics only. Typical motion equations of the satellite with faults are shown in [21]

$$
\begin{aligned}
& \dot{x}=f(x)+G(x) u+T_{d}+f_{a}(t) \\
& f(x)=\left[\begin{array}{c}
\frac{\cos \left(\theta_{3}\right) \omega_{2}-\sin \left(\theta_{3}\right) \omega_{3}}{\cos \left(\theta_{2}\right)}-\omega_{0} \\
\sin \left(\theta_{3}\right) \omega_{2}+\cos \left(\theta_{3}\right) \omega_{3} \\
\omega_{1}+\frac{\sin \left(\theta_{2}\right)}{\cos \left(\theta_{2}\right)}\left(\sin \left(\theta_{3}\right) \omega_{3}-\cos \left(\theta_{3}\right) \omega_{2}\right) \\
\frac{I_{2}-I_{3}}{I_{1}}\left(\omega_{2} \omega_{3}-3 \omega_{0}^{2} \zeta_{2} \zeta_{3}\right) \\
\frac{I_{3}-I_{1}}{I_{2}}\left(\omega_{3} \omega_{1}-3 \omega_{0}^{2} \zeta_{3} \zeta_{1}\right) \\
\frac{I_{1}-I_{2}}{I_{3}}\left(\omega_{1} \omega_{2}-3 \omega_{0}^{2} \zeta_{1} \zeta_{2}\right)
\end{array}\right] \\
& G(x)=\left[\begin{array}{ccc}
0 & 0 & 0 \\
0 & 0 & 0 \\
0 & 0 & 0 \\
1 / I_{1} & 0 & 0 \\
0 & 1 / I_{2} & 0 \\
0 & 0 & 1 / I_{3}
\end{array}\right] \quad T_{d}=\left[\begin{array}{c}
0 \\
0 \\
0 \\
T_{d 1} / I_{1} \\
T_{d 2} / I_{2} \\
T_{d 3} / I_{3}
\end{array}\right] \\
& \zeta=\left[\begin{array}{c}
-\sin \left(\theta_{1}\right) \cos \left(\theta_{2}\right) \\
\cos \left(\theta_{1}\right) \sin \left(\theta_{3}\right)+\sin \left(\theta_{1}\right) \sin \left(\theta_{2}\right) \cos \left(\theta_{3}\right) \\
\cos \left(\theta_{1}\right) \cos \left(\theta_{3}\right)-\sin \left(\theta_{1}\right) \sin \left(\theta_{2}\right) \sin \left(\theta_{3}\right)
\end{array}\right] \\
& f_{a}(t)=\left[f_{a 1}, f_{a 2}, f_{a 3}, f_{a 4}, f_{a 5}, f_{a 6}\right]^{\top}
\end{aligned}
$$

where $x=\left[\theta_{1}, \theta_{2}, \theta_{3}, \omega_{1}, \omega_{2}, \omega_{3}\right]^{\top}$ is the state vector, in which $\theta_{1}, \theta_{2}, \theta_{3}$ are the roll, pitch, and yaw angles about the body fixed coordinates. $\omega_{1}, \omega_{2}, \omega_{3}$ are the instantaneous angular velocities with respect to the fixed inertial space. $u=\left[T_{1}, T_{2}, T_{3}\right]^{\top}$ is the control torque vector and $T_{d}=$ $T_{m}+T_{a}$, sum of the worst-case aerodynamics and magnetic torques, represents the external disturbance vector. For lowEarth orbit (LEO) satellite below 1000-km altitude, gravitygradient torque, aerodynamic torque, and Earth magnetic torque are the primary environmental disturbances. $\omega_{0}$ denotes the orbital angular velocity of the mass center of the satellite. $I_{i}, i=1,2,3$ are the principal axis moments of inertia of the satellite. $f_{a i}$ is the possible faults occurred at the $i$ th state. The parameters of the microsatellite model in simulation are listed in Table I [22]. A variety of controller design methods have been proposed for the satellite attitude control, for example, $H_{\infty}$ control and nonlinear control, etc. Since the main goal of this study is to detect and identify faults, a sliding mode control method is adopted to stabilize the system.

In order to demonstrate the capability of the proposed neural adaptive observer, several kinds of actuator faults or system component faults are designed, including abrupt faults as well as incipient faults.

Using the methods in section III, a neural adaptive
TABLE I

MICROSATELLITE MAIN PARAMETERS

\begin{tabular}{|c||c|}
\hline Principal moment of inertia $I_{1}\left(\mathrm{~kg} \mathrm{~m}^{2}\right)$ & 18.4 \\
Principal moment of inertia $I_{2}\left(\mathrm{~kg} \mathrm{~m}^{2}\right)$ & 18.2 \\
Principal moment of inertia $I_{3}\left(\mathrm{~kg} \mathrm{~m}^{2}\right)$ & 6.8 \\
Orbital rate $\omega_{0}(\mathrm{rad} / \mathrm{s})$ & 0.0011 \\
Worst-case magnetic torque $T_{m}(\mathrm{~N} \mathrm{~m})$ & $5 \times 10^{-5}$ \\
Worst-case aerodynamic torque $T_{a}(\mathrm{~N} \mathrm{~m})$ & $1.56 \times 10^{-5}$ \\
\hline
\end{tabular}

observer is constructed as follows:

$$
\begin{aligned}
\dot{\hat{x}}(t) & =f(\hat{x})+G(x) u+M(t) \\
M_{i}(t) & =W_{i}(t) \sigma\left(\sum_{j=1}^{3} V_{i, j}(t) M_{i}(t-j \tau)+V_{i, 4}(t) \tilde{y}_{i}(t-\tau)\right)
\end{aligned}
$$

The choice of tapped delay $p$ and $q$ is based on a heuristic method or called trial-and-error method. The initial values of the parameters in the EKF updating law is $P_{i}(0)=10 I_{n}$ and $R_{i}(0)=2 \times 10^{-5}$. The default value of coefficient $\eta_{i}$ and initial values of parameters $\theta_{i}$ are 1 and 0.5 respectively. These parameters can be specifically adjusted in order to obtain a better performance.

The simulation results are shown from Fig.1 to Fig.3, from which several conclusions can be derived. First of all, when a fault occurs at a certain state, all the state estimation errors will be larger than a certain threshold and all the observer input $M_{i}(t)$ will react to estimate the errors. Hence, the state estimation errors and observer inputs can both be used to detect faults. However, only the observer input associated with the state where the fault occurs is nonzero after the fault occurs and other observer inputs return to zero or close to zero. In addition, the nonzero observer input approximates the fault quickly with high accuracy. Therefore, the observer inputs are not only able to detect and locate the fault, but also to specify its characteristics.

\section{CONCLUSIONS}

In this paper, a neural adaptive observer for nonlinear fault detection and identification is investigated. The NAO input is updated online via previous observer input and system output error by using the EKF algorithm. Under the guarantee of certain conditions, the proposed neural adaptive observer can detect and identify the deviation in the system caused by faults and disturbances. The application of this FDI scheme to a microsatellite attitude control system shows that the observer input can not only detect the faults, but also identify the characteristics of abrupt and incipient faults with good performance.

\section{Acknowledgement}

This research was supported in part by Canadian Space Agency (CSA) and Natural Sciences and Engineering Research Council (NSERC) of Canada. 

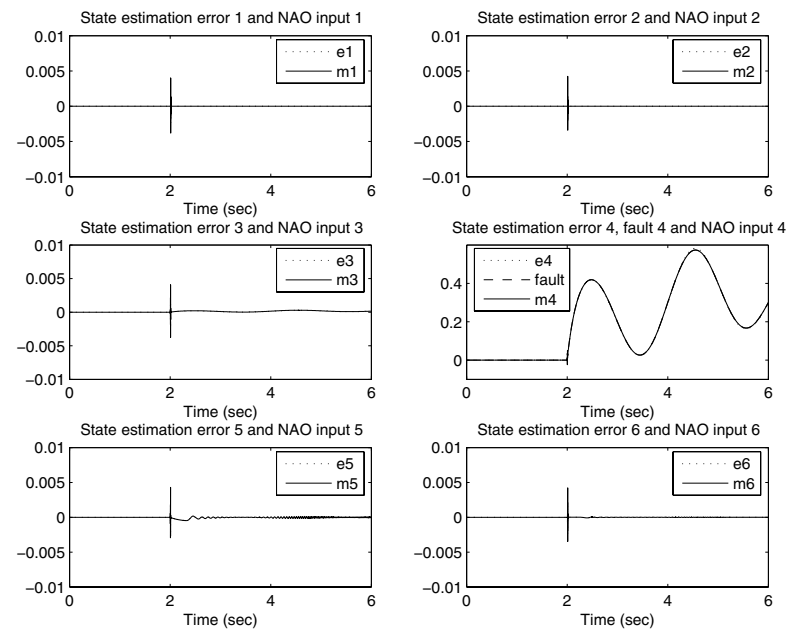

Fig. 1. State estimation errors and NAO inputs with fault 4
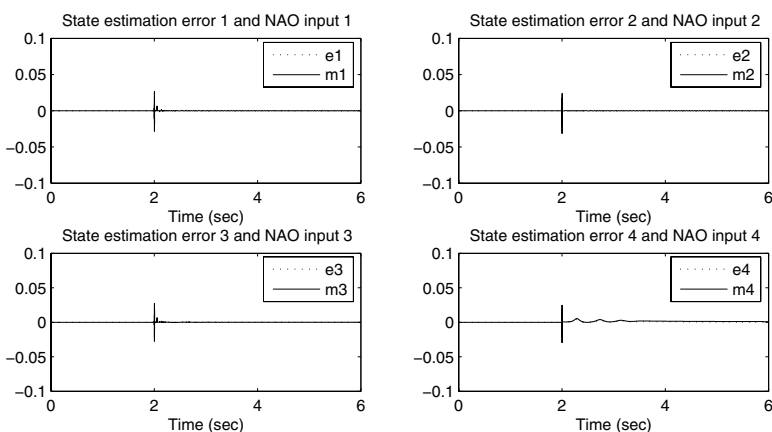

State estimation error 5 , fault 5 and NAO input 5
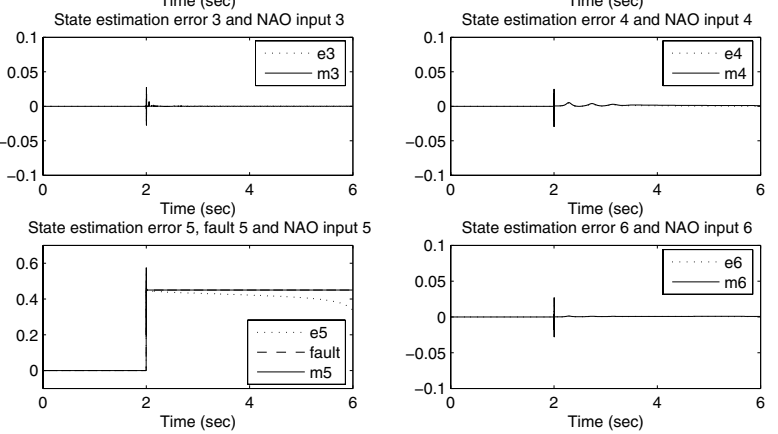

Fig. 2. State estimation errors and NAO inputs with fault 5
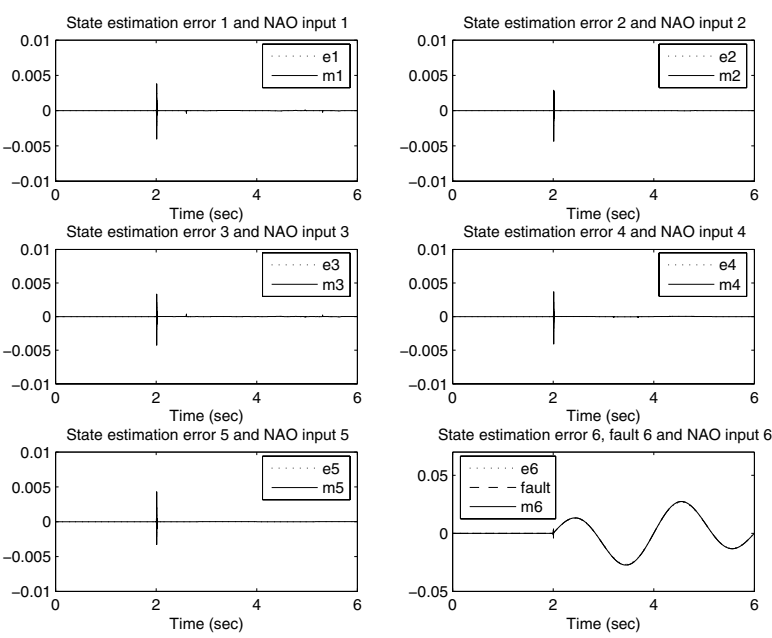

Fig. 3. State estimation errors and NAO inputs with fault 6

\section{REFERENCES}

[1] R.J. Patton, P.M. Frank and R.N. Clark, Fault Diagnosis in Dynamic Systems: Theory and Applications, Prentice-Hall, Englewood Cliffs, NJ; 1989.

[2] J. Chen and R.J. Patton, Robust Model-based Fault Diagnosis for Dynamic Systems, Kluwer Academic Publishers, Boston; 1999.

[3] V. Venkatasubramanian, R. Rengaswamy, K. Yin and S.N. Kavuri, A Review of Process Fault Detection and Diagnosis: Part I: Quantitative Model-based Methods, Computers and Chemical Engineering, vol. 27, 2003, pp. 293-311.

[4] V. Venkatasubramanian, R. Rengaswamy, K. Yin and S.N. Kavuri, A Review of Process Fault Detection and Diagnosis: Part II: Qualitative Models and Search Strategies, Computers and Chemical Engineering, vol. 27, 2003, pp. 313-326.

[5] V. Venkatasubramanian, R. Rengaswamy, K. Yin and S.N. Kavuri, A Review of Process Fault Detection and Diagnosis: Part III: Process History Based Methods, Computers and Chemical Engineering, vol. 27, 2003, pp 327-346.

[6] W. Chen and M. Saif, "An Iterative Learning Observer-based Approach to Fault Detection and Accommodation in Nonlnear Systems", in Proc. of 40th IEEE Conference on Decision and Control, Orlando, Florida, 2001, pp. 4469-4474.

[7] W. Chen and M. Saif, "Fault Detection and Accommodation in Nonlinear Time-delay Systems", in Proc. of American Control Conference, Denver, Colorado, 2003, pp. 4255-4260.

[8] S. Simani, C. Fantuzzi, and R.J. Patton, Model-based Fault Diagnosis in Dynamic Systems Using Identification Techniques, Springer, Berlin; 2003.

[9] K. Funahashi and Y. Nakamura, Approximtion of Dynamic Systems by Continunous Time Resurrent Neural Networks, Neural Networks, vol. 6, 1993, pp. 801-806.

[10] S. Naidu, E. Zafirou and T.J. Mcavoy, Use of Neural-Networks for Failure Detection in A Control System, IEEE Control Systems, vol. 10, 1990, pp. 49-55.

[11] T. Marcu, and L. Mirea, Robust Detection and Isolation of Process Faults Using Neural Networks, IEEE Control Systems, vol. 17, 1997, pp. $72-79$.

[12] T. Marcu, L. Mirea, P.M. Frank, "Neural Observer Schemes for Robust Detection and Isolation of Process Faults", in Proc. of UKACC International Conference on CONTROL'98, Swansea, UK, 1998, pp. 958-963.

[13] P.M. Frank and B. Koppen-Seliger, New Developments Using AI in Fault Diagnosis, Engineering Application of Artificial Intelligence, vol. 10, 1997, pp. 3-14.

[14] A. Alessandri and T. Parisini, "Neural State Estimators for Direct Model-based Fault Diagnosis", in Proc. of American Control Conference, Philadelphia, PA, 1998, pp. 2874-2878.

[15] R. Hecht-Nielsen, "Theory of the Backpropagation Neural Network", in Proc. of International Joint Conference on Neural Networks, vol. 1, 1989, pp. 593-605.

[16] G. Lera, and M. Pinzolas, Neighborhood Based Levenberg-Marquardt Algorithm for Neural Network Training, IEEE Transactions on Neural Networks, vol. 13, 2002, pp. 1200-1203.

[17] Y. Iiguni, H. Sakai and H. Tokumaru, A Real-time Learning Algorithm for A Multilayered Neural Network Based on the Extended Kalman Filter, IEEE Transactions on Signal Processing, vol. 40, 1992, pp. 959-966.

[18] L. Ljung and T. Söderström, Theory and Practice of Recursive Identification, MIT Press, Massachusetts; 1983.

[19] J.M. Zamarreno and P. Vega, State Space Neural Network, Properties and Application, Neural Networks. vol. 11, 1998, pp. 1099-1112.

[20] S. Barnett, Matrices in Control Theory, Nostrand Reinhold, New York; 1971.

[21] S.N. Singh and A. Iyer, "Nonlinear Decoupling Sliding Mode Control and Attitude Control of Spacecraft", IEEE Trans. on Aerospace and Electronic System, vol. 25, 1989, pp. 621-633.

[22] C.D. Yang, and Y.P. Sun, Mixed $H_{2} / H_{\infty}$ Sate-feedback Design for Micorsatellite Attitude Control, Control Engineering Practice, vol. 10, 2002, pp. 951-970. 